\title{
A MULTICENTRIC STUDY ON BREAST CANCER IN ULTRA YOUNG WOMEN: III - THERAPEUTIC ASPECTS AND ONCOLOGICAL OUTCOMES
}

Adriana Akemi Yoshimura ${ }^{1}$, André Mattar ${ }^{2}$, Bruna S. Mota ${ }^{3}$, Carlos Elias Fristachi ${ }^{4}$, Eduardo Carvalho Pessoa ${ }^{5}$, Felipe Eduardo Andrade ${ }^{6}$, Giuliano Tosello ${ }^{7}$, Heloísa Maria De Luca Vespoli5, Idam de Oliveira Junior ${ }^{8}$, João Bosco Ramos Borges ${ }^{9}$, José Roberto Filassi ${ }^{3}$, Luiz Henrique Gebrim¹, Marina Filie Haddad Piccinilli10, René Aloisio Costa Vieira ${ }^{8}$, Vicente Tarriconi Junior ${ }^{10}$, Alfredo Carlos Simões Dornellas de Barros

${ }^{1}$ Hospital da Beneficência Portuguesa - São Paulo (SP), Brazil.

${ }^{2}$ Hospital Pérola Byington-São Paulo (SP), Brazil.

${ }^{3}$ Instituto do Câncer do Estado de São Paulo - São Paulo (SP), Brazil.

${ }^{4}$ Instituto Arnaldo Vieira de Carvalho - São Paulo (SP), Brazil.

${ }^{5}$ Hospital das Clínicas de Botucatu - Botucatu (SP), Brazil.

${ }^{6}$ Hospital Sírio Libanês - São Paulo (SP), Brazil.

${ }^{7}$ Hospital Regional de Presidente Prudente - Presidente Prudente (SP), Brazil.

${ }^{8}$ Hospital do Amor-Barretos (SP), Brazil.

${ }^{9}$ Faculdade de Medicina de Jundiaí- Jundiaí (SP), Brazil.

${ }^{10}$ Faculdade de Ciências Médicas de Santos - Santos (SP), Brazil.

Introduction: We have originally introduced the concept of ultra young women (UYW), defined as age $\leq 30$ years old. It is generally accepted that UYW patients with breast cancer (BC) share some unfavorable outcomes and the patients are faced with family and professional problems, and unique quality of life issues, including loss of fertility, contraception, pregnancy, sexuality, cancer during pregnancy, body image and emotional distress, that complicate treatment decisions making. Objectives: Study the type of surgical and systemic treatment and oncologic outcomes in UYW with BC. Methods: We conducted a multicentric, observational, retrospective study of consecutive BC UYW patients. Only patients with infiltrating BC were included. Nine Mastology Centers located in the State of São Paulo participated. The following data were recorded: type of surgery, chemotherapy, endocrinetherapy, and radiotherapy. Individual oncologic evolution was analyzed and the patients were classified as alive without disease (AWD), alive with local recurrence (ALR), alive with systemic recurrence (ASR), died from BC (DBC) or died from another cause (DOC). The research protocol was approved by the Ethics Committee of all Collaborative Centers. Results: Sixteen percent of UYW with BC underwent mastectomies, $10 \%$ nipple-sparing mastectomies and $16 \%$ breast conservative surgeries. About $50 \%$ had immediated breast reconstruction. Sentinel node biopsy was performed in $24 \%$. $18 \%$ had more than four compromised LNs, $8 \%$ with extracapsular leak. $37 \%$ received adjuvant or palliative chemotherapy. $61 \%$ were submitted to irradiation. $54 \%$ had adjuvant hormonetherapy. The mean time of follow-up was 41.5 months (1.5-207). It was observed that $59 \%$ were AWD, $1 \%$ ALR, $7 \%$ ASR and $23 \%$ $\mathrm{DBC}$, unfortunately standing out the elevated contingent of BC-related deaths. Conclusions: BC therapy in UYW were tailored according to individual characteristics, but the oncological outcomes in this age range at the moment could be considered unfavorable. 